\title{
Rotating Anisotropic Crystalline Silicon Nanoclusters in
}

\section{Graphene}

Qu Chen, ${ }^{1}$ Ai Leen Koh, ${ }^{2}$ Alex Robertson, ${ }^{l}$ Kuang He, ${ }^{l}$ Gun-Do-Lee, ${ }^{3}$ Robert Sinclair ${ }^{4}$, Jamie H. Warner $^{1 *}$

${ }^{1}$ Department of Materials, University of Oxford, Parks Road, Oxford, OX1 3PH, UK

${ }^{2}$ Stanford Nano Shared Facilities, Stanford University, Stanford, CA 94305-4045, USA

${ }^{3}$ Department of Materials Science and Engineering, Seoul National University, Seoul 151-742,

Republic of Korea

${ }^{4}$ Department of Materials Science and Engineering, Stanford University, Stanford, CA

94305-4034, USA

*Jamie.Warner@materials.ox.ac.uk

\begin{abstract}
The atomic structure and dynamics of silicon nanoclusters covalently bonded to graphene are studied using aberration-corrected transmission electron microscopy. We show that as the cluster size increases to 4-10 atoms, ordered crystalline cubic phases start to emerge. Anisotropic crystals are formed due to higher stability of the $\mathrm{Si}-\mathrm{C}$ bond under electron beam irradiation compared to the Si-Si bond. Dynamics of the anisotropic crystalline Si nanoclusters reveals they can rotate perpendicular to the graphene plane, with oscillations between the two geometric configurations driven by local volume constraints. These results provide important insights into the crystalline phases of clusters of inorganic dopants in graphene at the intermediate size range between isolated single atoms and larger bulk 2D forms.
\end{abstract}


KEYWORDS: graphene, TEM, dopants, $\mathrm{Si}$, atoms.

Introducing foreign dopant atoms to pristine graphene provides an opportunity to manipulate the key properties, such as carrier density, ${ }^{1,2}$ band structure, ${ }^{3,4}$ and magnetism, ${ }^{5}$ which further broaden the practical applications of graphene in nano-electronic applications including semiconductor devices. ${ }^{6,7}$ Impurity atoms can be incorporated as either surface adatoms, ${ }^{8}$ or as dopants covalently bonded within the graphene lattice. Covalently bonded dopants in graphene are more robust and have been explored with a variety of elements, such as $\mathrm{N},{ }^{9} \mathrm{Si},{ }^{10} \mathrm{Fe},{ }^{11,12}$ and $\mathrm{Cu} .{ }^{12}$ Several forms of impurity dopants have been studied from single atoms to larger 2D membranes containing hundreds of atoms. Robertson et al. reported the dynamics of single Fe atoms in monovacancies and divacancies, where nanoscale control of dopant incorporation was achieved by the use of controlled electron beam irradiation to create the initial vacancy that capture the mobile Fe atoms. ${ }^{13}$ The single Fe dopants in graphene act as sites for trapping additional Fe atoms, leading to Fe dimer formation in graphene. ${ }^{13}$ The Fe dimers were found to be mostly magnetic, and offer a way of introducing magnetism into doped graphene. ${ }^{14}$ Single Si dopants in graphene induce plasmonic enhancement when embedded in the monolayer graphene lattice, which may play a role in future applications in plasmonic devices. ${ }^{15}$ The research interest of small clusters of impurity atoms in graphene has led to the direct observation of Si trimers and $\mathrm{Si}_{6}$ clusters. ${ }^{10,16}$ Under the electron beam, both small clusters of $\mathrm{Si}$ exhibited dynamics associated with structural reconfigurations, along with periods of fixed stability needed for image acquisition. ${ }^{10,16}$ Monolayer $2 \mathrm{D}$ nanoscale regions containing tens and hundreds of Fe atoms suspended within graphene nanopores were recently observed. ${ }^{17}$

Introduction of impurity dopants into graphene lattice with precise spatial control is challenging, and valid methods have been used including chemical modification ${ }^{18,19}$, intercalation, ${ }^{20,21}$ low 
energy ion implantation, ${ }^{22}$ and defect-assisted doping by electron beam radiation. ${ }^{13,14}$ The last method, which involves in the generation of vacancies or small holes via focused electron beam irradiation from an aberration-corrected transmission electron microscope (AC-TEM) with an accelerating voltage of $80 \mathrm{kV}$, is a promising technique for manipulating dopants at the nanoscale. ${ }^{23}$ The created defects exhibit enhanced chemical reactivity, which provides the capability of trapping highly mobile adatoms residing on the graphene surface. ${ }^{13}$ Silicon is one of the most commonly seen dopants in graphene as it can be originated from quartz tubes used during chemical vapour deposition (CVD) growth, glass beakers, $\mathrm{Si}$ substrates and $\mathrm{Si}_{3} \mathrm{~N}_{4}$ TEM grids during both graphene growth and the transfer procedure. The atomic structure and dynamics of both graphene and its individual dopants can be captured using aberration-corrected transmission electron microscopy (AC-TEM), using low accelerating voltages of $<80 \mathrm{kV} .{ }^{24-26}$

The prior atomic resolution studies of covalently bonded dopants in graphene by AC-TEM have focused primarily on either the very small clusters (1-6 atoms) or the larger 2D membranes, with little work performed within the intermediate size regime of small nanoclusters. For large numbers of atoms, crystalline periodic lattice structures will form, whilst in very small dopant clusters, such as trimers, there are not enough atoms present to form stable crystalline forms. In this report we examine the atomic structure and time-dependent dynamics of $\mathrm{Si}$ nanoclusters that contain sufficient numbers of atoms to begin to show the initial stages of periodic ordering. The energy transferred from the electron beam to the graphene specimen can trigger the movement of impurity atoms, allowing time-dependent behavior to be studied. ${ }^{13}$ Throughout the graphene sample we observed several regions containing Si dopants covalently bonded in the lattice with cluster sizes ranging from 1-10, as shown in figure 1. Energy dispersive $\mathrm{X}$-ray spectroscopy was measured from suspended regions of graphene containing patches of surface amorphous carbon that included 
heavier inorganic elements and identified as $\mathrm{Si}$ (see supporting information figure $\mathrm{S} 1$ ). Some areas contained a high density of Si dopants clusters with variable atomic number, such as the area shown in figure 1(e), and within this area we can locate several single Si atom dopants and dimers (yellow dashed circles) as well as Si nanoclusters with atomic numbers up to 10 or more (white dashed circles). Our focus of this study is the structure of these Si nanoclusters and their dynamics.
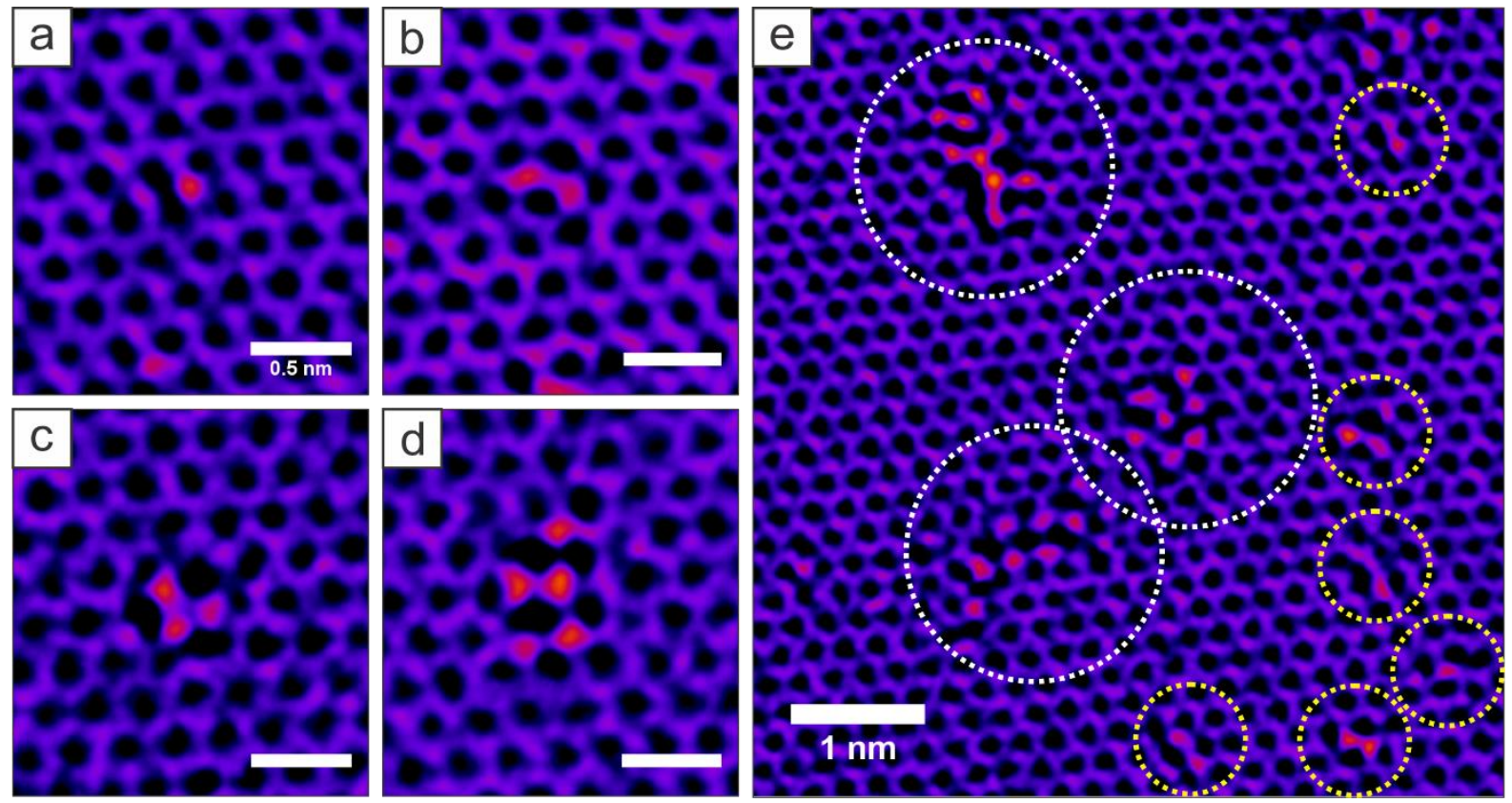

Figure 1. AC-TEM images showing examples of increasing cluster sizes of Si dopants in graphene ranging from (a) one, (b) two, (c) three, (d) five, and (e) a region of heavily doped graphene with multiple Si nanoclusters. White dashed circle indicates nanoclusters, yellow dashed circle indicates atomic dopant and dimers.

\section{Results and Discussion}


Under continuous electron beam irradiation at $80 \mathrm{kV}$, the small Si nanoclusters exhibit dynamic structural reconfigurations, but in many cases were stable enough in a cubic lattice configuration to obtain images with 2 second acquisition times. Figure $2 \mathrm{i}$ shows several examples from different regions of the sample of Si nanoclusters with cubic arrangement of the atoms. A schematic atomic model of how the Si atoms can form such cubic arrangements is presented in figure $2 \mathrm{i}(\mathrm{f})$. The cubic packing typically consists of pairs of $\mathrm{Si}$ atoms joined together to form anisotropic crystals, such as in figure $2 \mathrm{i}(\mathrm{b})$. It is known that $\mathrm{Si}$ can adopt two different oxidation states when bonding in graphene, resulting in connection to either three or four carbon atoms. Within the anisotropic crystals in figure $2 \mathrm{i}$, the $\mathrm{Si}$ atoms are all bonded to at least one $\mathrm{C}$ atom, and the $\mathrm{Si}$ atoms that are not at the ends of the crystal are bonded to three nearest neighbor Si atoms. Non-hexagonal carbon rings (i.e pentagons and heptagons) around the Si cluster cause deviation from exact symmetry in these structures. The anisotropic nanocrystals were stable for long enough to capture dynamics of the crystal lattice.

Figure 2ii shows the formation of an ordered $\mathrm{Si}$ cluster from a group of randomly located $\mathrm{Si}$ atoms. The $\mathrm{Si}$ atoms are restricted to a small area, as shown in figure 2ii(a) and 2ii(b), and some Si atoms are even distorted out-of plane due to the limited space provided by bulk graphene lattice. This disordered $\mathrm{Si}$ cluster is unstable and the rearrangement of $\mathrm{Si}$ atoms happens within $11 \mathrm{~s}$. This restructuring is the consequence of the expansion of the local area, as three $\mathrm{C}$ atoms highlighted in figure 2ii(b) are sputtered out by electron beam radiation. The sufficient space induces the formation of ordered $\mathrm{Si}$ cluster shown in figure $2 \mathrm{ii}(\mathrm{d})$. The $\mathrm{C}$ atoms connected to the $\mathrm{Si}$ cluster also adjust themselves to generate zigzag conformation. The two 5-atoms rings may generated from Stone-Wales rotation of two $\mathrm{C}$ atoms along zigzag edge, as shown in the red rectangle in figure 2ii(e), with the red dashed circles representing the position of $\mathrm{C}$ atoms before SW rotation. The 
behavior of Si atoms fitting themselves into existing ordered Si cluster is observed in figure 2ii(d) and 2ii(g), and their atomic models (figure 2ii(e) and 2ii(h)) show this phenomenon more clearly. The two Si atoms in the red doted box in figure 2e move downwards as an integrity, becoming a part of the ordered structure. This movement is along with the rearrangement of $\mathrm{C}$ atoms, which prolong the zigzag edge around the Si cluster.

We observed that the anisotropic Si nanoclusters would rotate such that two $\mathrm{Si}$ atoms were in projection. Figure 3 shows an ordered Si nanocluster observed in AC-TEM images (a, d) as well as the multislice image simulations (c, f) based on the atomic models (b, e). Two Si atoms marked by yellow dashed ellipse in figure 3a rotate by 90 degrees, forming out-of-plane stacking structure (yellow dashed circle in figure 3d) within 8 s. To prove the stacking of the Si structure, boxaveraged intensity profiles taken from both AC-TEM and simulation images are presented in figure S2 in Supporting Information. The evaluation method for distinguishing the different number of $\mathrm{Si}$ atoms in projection is the ratio of intensities: $\frac{I_{\text {Stacking } S i-I_{C}}}{I_{\text {Single } S i}-I_{C}}$. Subtracting intensity of carbon from both stacked Si and single Si projections eliminates the influence of background levels. For ACTEM image, the value is 3.52 , whilst for the simulated image, the value is 3.67 , providing an excellent match. . This confirms the pair of Si atoms can rotate perpendicular to the graphene plane to form an out-of-plane structure with likely each $\mathrm{Si}$ atom on either side of the graphene plane. 

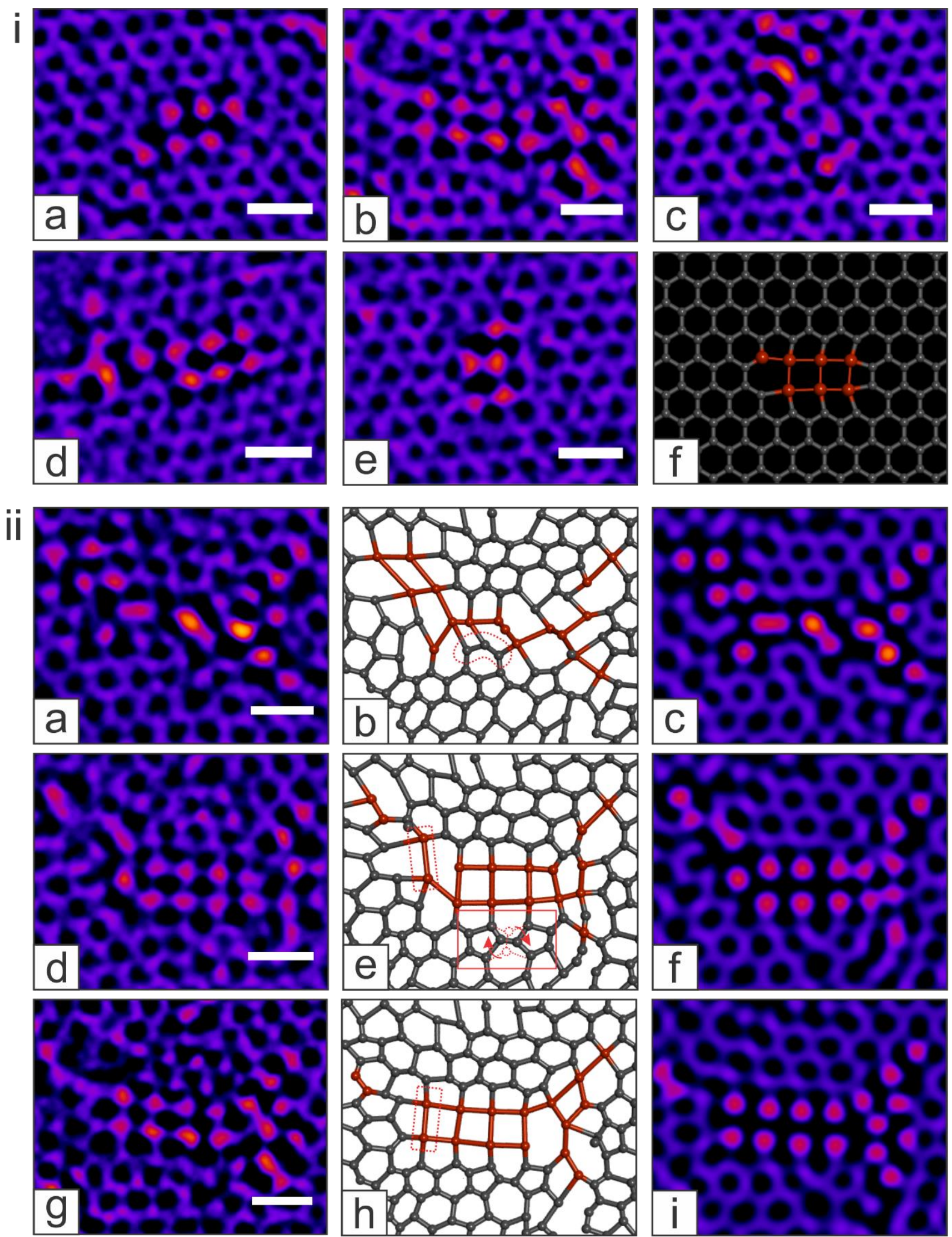

Figure 2. i (a)-(e) AC-TEM images of various Si clusters in graphene from different regions of the sample showing similar cubic structural forms. (f) Atomic model illustrating Si atoms covalently bonded in 
graphene adopting a cubic structure. ii (a) AC-TEM image of Si cluster with randomly located Si atoms. (b) The atomic model of (a), red ones are for Si atoms. (c) Multislice image simulation based on (b). (d) AC-TEM image of the same Si cluster captured after 11 s. (e) The atomic model of (d). (f) Multislice image simulation based on (e). (g) AC-TEM image taken after 19 s. (h) The atomic model of (g). (i) Multislice image simulation based on (h). Red dotted closed curves in ii(b) highlights the $\mathrm{C}$ atoms which are sputtered out. Scale bar: $0.5 \mathrm{~nm}$.
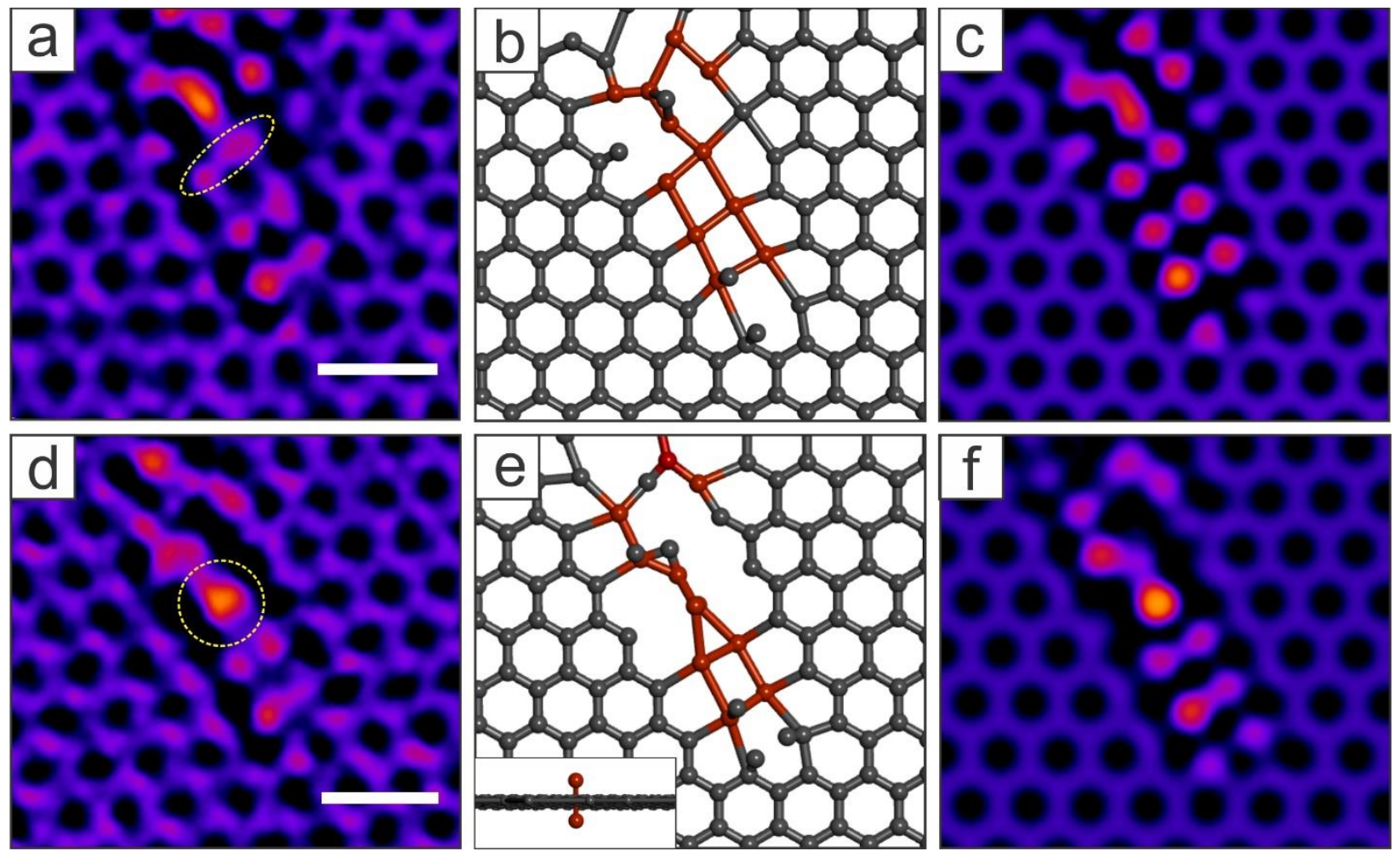

Figure 3. The ordered structure of a Si nanocluster and the out-of-plane rotation of two Si atoms. a)

A small Si nanocluster showing ordered cubic phases. The yellow dashed ellipse indicates the region where rotation will happen. b) The atomic model of a). c) Multislice image simulation based on the atomic model in b). d) The rotation of two Si atoms showing a stacked structure, highlighted in yellow dashed circle. The time duration between images in d) and a) is $8 \mathrm{~s}$. e) The atomic model of d), with the side view of the stacked Si atoms shown in the inset. f) Multislice image simulation based on e). Scale bar: $0.5 \mathrm{~nm}$. 
The specific details of the dynamics of the $\mathrm{Si}$ atom rotation and local bonding changes are examined in figure 4 , which are figure $3 \mathrm{a}$ and $3 \mathrm{~d}$ rotated clockwise by $37.5^{\circ}$ with bonds highlighted by lines. A Klein edge, circled in white dashed circle in figure 4a, is initially observed and then disappeared by the next image in figure $4 \mathrm{~b}$, and two additional $\mathrm{C}$ atoms (in white circles) joined in two 5-atom rings, forming two 6-atoms rings. The additional $\mathrm{C}$ atoms may either come from the missing Klein edge or other highly mobile $\mathrm{C}$ atoms sputtered from elsewhere by electron beam irradiation. The rearrangement of $\mathrm{C}$ atoms indicates that the edge around the ordered $\mathrm{Si}$ cluster tends to adopt zigzag conformation, which consequently restricts the space where the two upper $\mathrm{Si}$ atoms occupy. The two $\mathrm{Si}$ atoms are rotated out of plane to compensate this shrinkage of space. The distance between the spots of contrast associated with $\mathrm{Si}-\mathrm{Si}$ and $\mathrm{Si}-\mathrm{C}$ bonds are measured to obtain more insights of before and after rotation.

The distances between two contrast spots from Si atoms before rotation are 2.05, 2.00, and 2.28 $\AA$, but the actual Si-Si bond lengths may differ because the TEM image is a 2D projection of the nanocluster, where out-of-plane distortion is involved. To further explore the bond distances within the ordered cluster when taking out-of-plane distortions into consideration, we utilize density functional theory (DFT) calculations. The atomic model for the DFT calculations have been simplified to eliminate irrelevant carbon adatoms and redundant $\mathrm{Si}$ atoms, as shown in figure 4c. The three Si-Si bonds are 3.29, 2.38 and $3.00 \AA$, from top to bottom, determined by the DFT model, and the corresponding bond lengths measured from the multislice image simulation (figure 4d) are 2.38, 2.31, $2.94 \AA$. The biggest difference emerges at the top Si pair, which is highlighted by blue spots, which is attributed to the out-of-plane structure. A side view of the atomic model from the direction indicated by the yellow arrow in figure $4 \mathrm{c}$ is presented in figure $4 \mathrm{e}$. The angle between the blue Si pair and graphene lattice is $45^{\circ}$, giving a shortened projected view of the Si- 
Si bond of $2.33 \AA$ A. Measurement of this distance from the image simulation gives a similar result. Figure 4f demonstrates the variation of relative energy with the increasing the rotation angle of this Si pair. A pure in-plane configuration is not energetically favourable due to the limited space and large $\mathrm{Si}-\mathrm{C}$ bond length distance, and the energy is lowest from $40^{\circ}$ to $50^{\circ}$, indicating the most stable structure corresponding to the DFT model shown in figure $4 \mathrm{c}$. The energy of $90^{\circ}$, namely, the two $\mathrm{Si}$ atoms forming a stacked structure as in figure $3 \mathrm{~d}$ and $4 \mathrm{~b}$, is about $3.5 \mathrm{eV}$ higher than the most stable state. This energy barrier is larger than the thermal contributions at room temperature, but well within the energy delivered by the electron beam. The other two Si pairs in the 6-atom nanocluster have less out-of-plane rotation, as $\mathrm{Si}$ atoms located at the end of the anisotropic crystals are exposed to more carbon atoms than those within the middle and are less able to rotate due to the $\mathrm{Si}-\mathrm{C}$ bond. The $\mathrm{Si}$ pair highlighted in blue possesses the highest degree of freedom in the cluster, resulting in out-of-plane rotating with a wide angle range. 

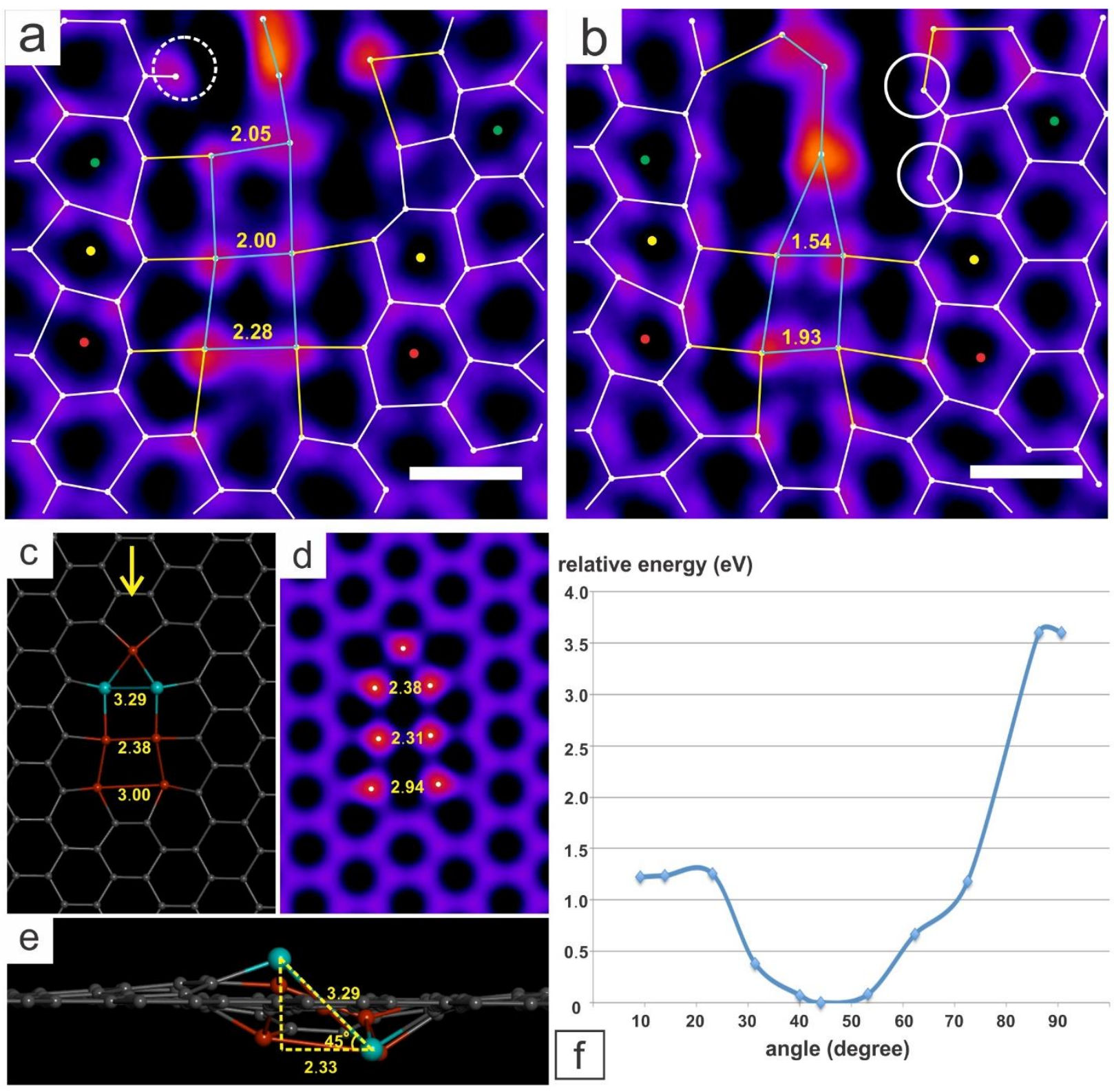

Figure 4. The bond lengths of ordered Si before a) and after b) rotation, with white lines for C-C bond, yellow lines for Si-C bond, and blue lines for Si-Si bond. The white dashed circle in a) shows a Klein edge that is missing in $\mathrm{b}$ ). The white circles in $\mathrm{b}$ ) show two additional $\mathrm{C}$ atoms contributing to form 6 -atom rings. c) DFT calculated atomic model for a 6-atoms ordered Si nanocluster (simplified from the model in figure 3b) with calculated Si-Si bond lengths. d) Multislice image simulation based on c) with bond lengths determined by the simulation. e) Lateral view of c) from the direction pointed by the yellow arrow. f) 
Relative energy variation against the rotation angle of the highlighted Si pair in blue. The bond lengths are in $\AA$. Scale bar: $3 \AA$.

The bulk graphene lattice surrounding the ordered Si cluster showed surprising stability with no measureable expansion or contraction after $\mathrm{Si}$ atoms rotation. This can be confirmed by measuring the lengths between the centers of 6-atoms rings with the same colour dots (red, yellow and green) shown in figure $4 \mathrm{a}$ and $4 \mathrm{~b}$. The distances between the red, yellow and green spots are $0.85,0.85$ and $1.03 \mathrm{~nm}$, respectively, both before and after the rotated $\mathrm{Si}$ atoms. These three values match the theoretical values for bulk graphene when no foreign atom is involved. It indicates that the pair of rotated stacked $\mathrm{Si}$ atoms is unlikely to be bonded to the neighboring $\mathrm{C}$ atoms as this covalent bond would cause structural distortions to the local graphene lattice. This is likely compensated by more bonding to neighbor $\mathrm{Si}$ atoms.

Figure 5 shows the inverse behavior in comparison to figures 3 and 4. Six Si atoms showing three out-of-plane stacking structures rotate to two-row crystalline alignment within $10 \mathrm{~s}$. The stacked $\mathrm{Si}$ atoms are shown in the inset of figure $5 \mathrm{~b}$, where three pairs of $\mathrm{Si}$ atoms are perpendicular to the graphene lattice. The stacked structures are also confirmed by box-average intensity profiles (see supporting information figure S3). A plausible route based on the two AC-TEM images is provided from figure $5 \mathrm{~g}$ to $5 \mathrm{j}$. The change starts with a missing carbon atom bonded with a $\mathrm{Si}$ and two $\mathrm{C}$ atoms, highlighted by a red dashed circle in figure $5 \mathrm{~b}$. The small cluster with two Si and a $\mathrm{C}$ atom shift upwards to fill the vacancy, making the $\mathrm{C}$ atoms below rearrange to adopt a zigzag conformation (figure 5g). This restructuring of $\mathrm{Si}$ and $\mathrm{C}$ atoms provides sufficient space for stacking atoms to relax (figure 5h). The rotation may happen one-by-one from left to right. Figure 5i shows slight adjustment mainly on the migration of $\mathrm{C}$ atoms from elsewhere to form more stable structures, as highlighted by the red circles. It can be seen that the adjustment only takes place 
around relatively free $\mathrm{Si}$ atoms, whereas when the $\mathrm{Si}$ atoms bond to more $\mathrm{C}$ atoms in the graphene lattice, the structure is stable, for example, the three $\mathrm{Si}$ atoms at the top end of the Si cluster. Three additional Si atoms come into the Si cluster in figure $5 \mathrm{j}$ (pointed by red arrow). The addition of $\mathrm{C}$ and $\mathrm{Si}$ atoms indicates that this $\mathrm{Si}$ cluster area exhibits enhanced chemical reactivity, capable to trap highly mobile adatoms moving along the surface of graphene. In this complicated Si cluster structure, most of the Si atoms are observed to have $\mathrm{sp}^{3}$ hybridization, as well as some with three $\mathrm{sp}^{2}$ bonds, and one bridging between two $\mathrm{Si}$ atoms forming a dangling bond.

This expansion of the Si cluster, involving stacked $\mathrm{Si}$ atoms relaxing as well as additional $\mathrm{Si}$ atoms joining in, does not influence the surrounding graphene structure. The distances between the same 6-atom rings (marked by red and yellow dots in figure 5a and 5d), have the same values before and after the reshaping of the Si cluster. The length between the two 6-atoms rings marked by red dots is $0.69 \mathrm{~nm}$, and the two $\mathrm{Si}$ atoms between them are $0.15 \mathrm{~nm}$ apart after relaxation.

The behavior of an ordered Si cluster is affected by its location, especially when it is adjacent to a hole. Figure 6 provides a good example to compare the dynamics between two 6-Si-atom clusters, one (left one in figure 6a) is close to a hole, and the other one (right one in figure 6a) is located in the bulk graphene lattice. It can be seen that after $49 \mathrm{~s}$, the left Si cluster loses its ordered structure by atoms rearrangement, while no obvious change is observed in the right $\mathrm{Si}$ cluster. The reshaping of the Si cluster adjacent to the hole is attributed to the higher mobility of edge atoms, meaning lower energy required to displace them. A plausible pathway for the cluster restructuring is shown from figure $6 \mathrm{~g}$ to $6 \mathrm{i}$. The hole is expanded due to the two $\mathrm{Si}$ atoms displacing the adjacent $\mathrm{C}$ atoms (figure $6 \mathrm{~b}$ and $6 \mathrm{~g}$ ), and the unstable dangling bonds and Klein edges are removed by continuous electron beam radiation (figure $6 \mathrm{~h}$ ). Three $\mathrm{C}$ atoms between two Si clusters (red doted region in figure $6 \mathrm{~g}$ ) are also removed, due to breaking the $\mathrm{Si}-\mathrm{C}$ bonding. A Si atom moves into the vacancy 
left by the removed $\mathrm{C}$ atoms, combining with the larger existing ordered Si structure. Figure 6i shows five $\mathrm{C}$ atoms migrate into the lattice to form 6-atom-rings, inducing the relocation of $\mathrm{Si}$ atoms. The additional $\mathrm{C}$ atoms probably come from the nearby sputtered $\mathrm{C}$ atoms, indicating that the expansion of a hole is the combined effect of atomic elimination and reconstruction. The restructuring of $\mathrm{C}$ atoms also takes place at the boundary of the Si cluster and graphene lattice, as shown in figure 6i. A C atom moves downwards, contributing to the formation of complete zigzag edge. This agrees well with the findings in figure 3 and figure 4 .
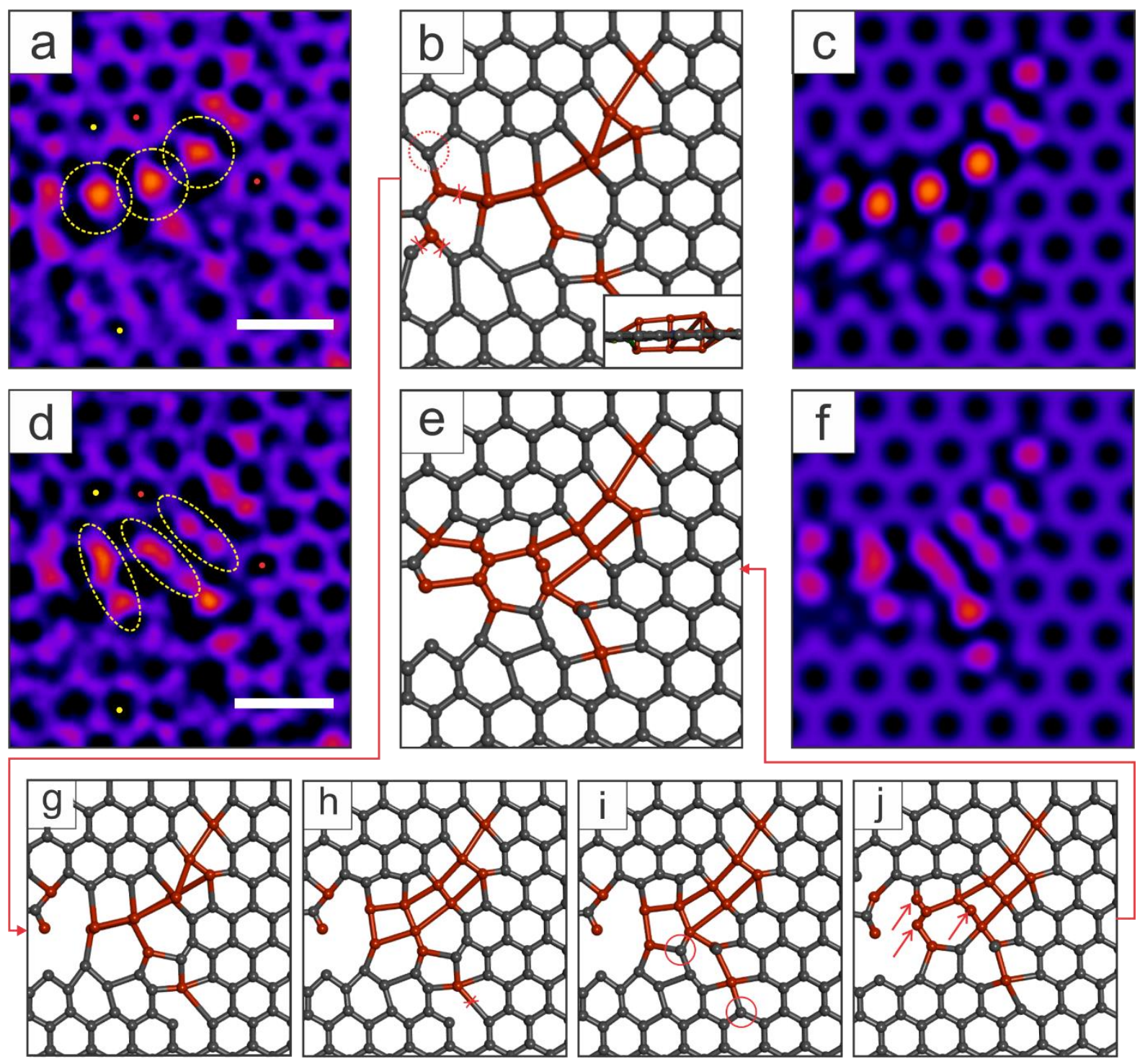
Figure 5. A Si cluster showing the rotation of Si atoms. a) AC-TEM images of a Si cluster, with the yellow dashed rings circling the stacking Si atoms. b) The atomic model of a), with the inset showing the lateral view of the stacking structure. c) Multislice image simulation based on b). d) AC-TEM image taken $10 \mathrm{~s}$ after a). The yellow dashed ellipses highlight the rotated Si atoms. e) The atomic model of d). f) Multislice image simulation based on d). g-j) A plausible dynamics for the structure alteration. Red dashed ring indicates the missing $\mathrm{C}$ atom in the following frame, red circle indicates the additional $\mathrm{C}$ atom, red arrows are for additional Si atoms, and crosses are for breaking bonds. Scale bar: $0.5 \mathrm{~nm}$.

In comparison with the 6-Si-atom ordered cluster in figure 3a, the Si cluster (right) in figure $6 \mathrm{~d}$ has elongated bond lengths, as shown in figure S4 in Supporting Information. The average Si-Si bond length is $2.54 \AA$, which is longer than the theoretical value of $\mathrm{Si}$-Si bond. It indicates that the Si atoms are likely sitting planar in the graphene lattice without significant out of plane tilting. The graphene lattice around the Si cluster, as a consequence, is pushed away from its original location. The distances between the centers of the 6-atom rings highlighted by red and yellow dots in figure $6 \mathrm{~d}$, are $0.92 \mathrm{~nm}$, which is nearly $10 \%$ longer than bulk graphene when no foreign atom is involved. The hole nearby the Si cluster probably contributes to the expanded structure, which is not observed in the former two Si clusters shown in figure 3 and figure 5, where both the Si clusters are located in the bulk graphene lattice. Therefore, when the surrounding graphene lattice is less rigid, the ordered Si cluster can adopt an in-plane structure.

The ordered Si clusters observed, no matter how many atoms involved, are in two rows, which means any $\mathrm{Si}$ atom within the ordered structure is connected to at least one $\mathrm{C}$ atom. More specifically, the $\mathrm{Si}$ atoms at the ends of a row form four $\mathrm{sp}^{3}$ bonds with two $\mathrm{C}$ atoms and two $\mathrm{Si}$ atoms, while the ones at the middle section constitute $\mathrm{sp}^{3}$ bonds with three $\mathrm{Si}$ atoms and a $\mathrm{C}$ atom. The reason why no Si atom with four Si neighbors is observed is attributed to the lower Si-Si bond 
energy than both $\mathrm{Si}-\mathrm{C}$ and $\mathrm{C}-\mathrm{C}$ bond as the covalent bond energy is inversely proportional to the atomic radii. The energy transferred from electron beam irradiation is higher than the threshold energy for knocking Si atoms with four adjacent Si atoms out of graphene lattice. Therefore, an ordered Si cluster with three or more rows is not able to stably exist under continuous electron beam irradiation at $80 \mathrm{kV}$ accelerating voltage.
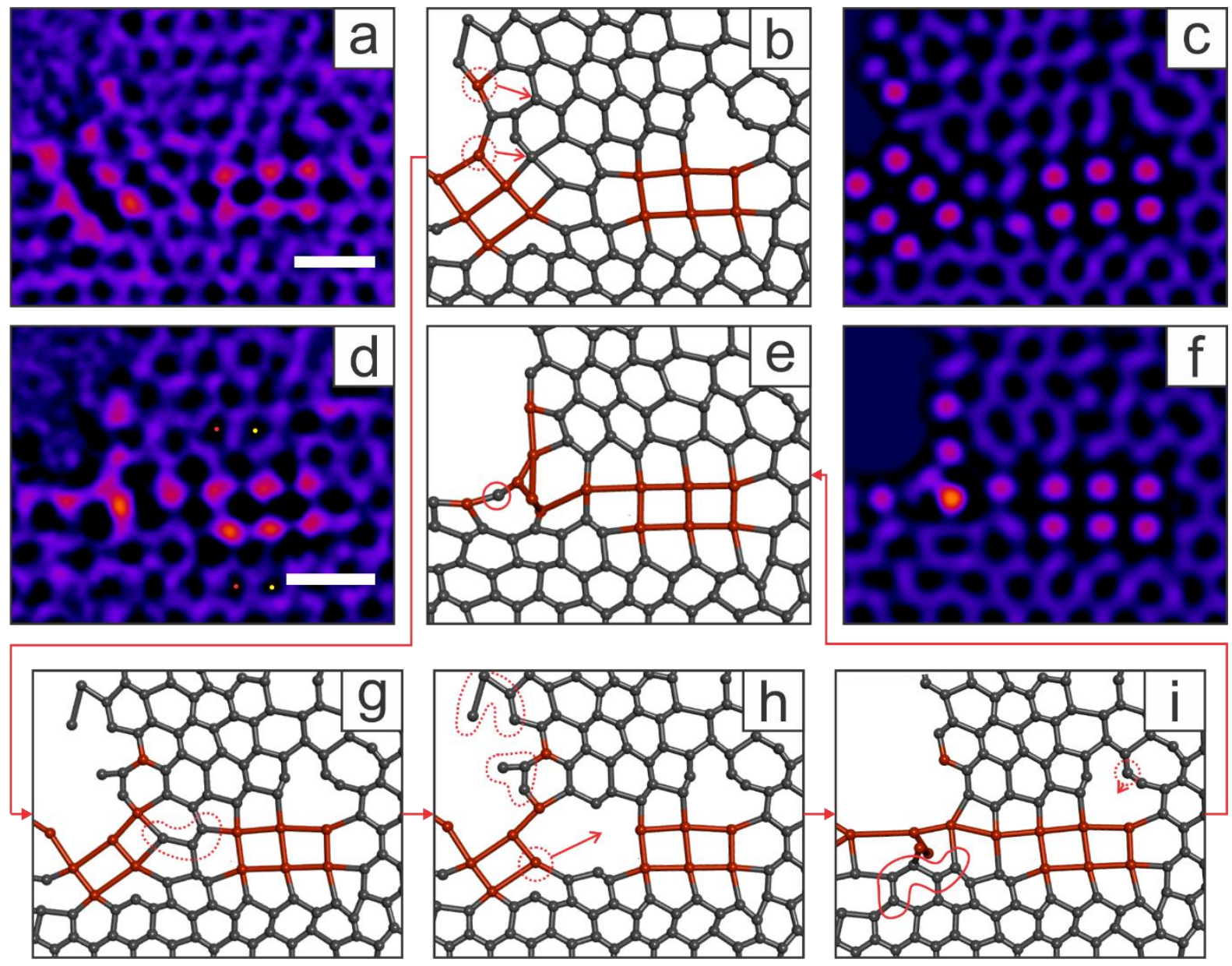

Figure 6 The restructure of a ordered Si cluster adjacent to a hole. a) AC-TEM image of two ordered

Si cluster, with the left one adjacent to a hole and the right one settled in graphene lattice. b) The atomic model of a). c) Multislice image simulation based on b). d) AC-TEM image taken $49 \mathrm{~s}$ apart showing the restructure of the left Si cluster. e) The atomic model of d). f) Multislice image simulation based on e). g-i) A plausible route for the reconstruction. Atoms circled by red dashed closed curves are either sputtered out 
or moved to contribute to other structures, and the ones in red solid closed curves are the additional atoms which are not in the positions in former images. Scale bar: $0.5 \mathrm{~nm}$.

\section{Conclusion}

We have observed crystalline anisotropic Si nanoclusters residing in graphene lattice. These clusters contain six to ten $\mathrm{Si}$ atoms, which line up in two rows to achieve stable morphology in electron beam irradiation environment. The clusters with ordered structure are observed to evolve from randomly located $\mathrm{Si}$ atoms when the space occupied by the cluster is enlarged by the elimination of several adjacent $\mathrm{C}$ atoms. The lowest energy configuration for a crystalline $\mathrm{Si}$ cluster based on DFT is not planar, but with the freest Si pair possesses an angle from $40^{\circ}$ to $50^{\circ}$ to the graphene sheet. An ordered Si cluster also tends to expand its volume by attracting surrounding Si atoms to join into the structure. Out-of plane rotation of two adjacent Si atoms and the inverse process were also observed in ordered Si clusters, and the energy barrier for an out-ofplane rotation is about $3.5 \mathrm{eV}$, confirming the possibility of the observation in TEM.

\section{Methods}

Synthesis and Transfer of Graphene. Graphene was synthesized on the surface of molten copper sheet, which acted as the catalyst, by chemical vapour deposition (CVD) in atmosphere pressure using previously reported methods ${ }^{27}$. A $1 \times 1 \mathrm{~cm}^{2}$ highly pure copper foil (Alfa Aesar, Puratonic $99.999 \%$ purity) with the thickness of $0.1 \mathrm{~mm}$ was placed on the top of a tungsten sheet with same size (Alfa Aesar, 99.95\% purity, $0.05 \mathrm{~mm}$ thickness), positioned in a furnace and then annealed at $1090^{\circ} \mathrm{C}$ for 30 min with a mixture gas flow of $200 \mathrm{sccm}$ pure Argon and $100 \mathrm{sccm} \mathrm{H}_{2}(20 \%) / \mathrm{Ar}$. For graphene growing, the $\mathrm{H}_{2} / \mathrm{Ar}$ was then decreased to $80 \mathrm{sccm}$, with the addition of $\mathrm{CH}_{4}(1 \%) / \mathrm{Ar}$ to the system for $90 \mathrm{~min}$. The sample was then cooled down to room temperature under a $\mathrm{H} 2 / \mathrm{Ar}$ 
atmosphere. A PMMA supporting scaffold was coated on the graphene sample by spin coating. The sample has a four-layer structure as tungsten/copper/graphene/PMMA from bottom to top. The tungsten layer was etched by an electrochemical reaction at $40^{\circ} \mathrm{C}$, with $2 \mathrm{~mol} / \mathrm{L} \mathrm{NaOH}$ solution as the electrolyte, tungsten as the anode, and a piece of copper foil (Alfa Aesar, 99.8\% purity, annealed, $0.025 \mathrm{~mm}$ thickness) as the cathode, as previously reported. ${ }^{28}$ The copper layer was then etched by $0.2 \mathrm{~mol} / \mathrm{L}$ ammonium persulfate solution, leaving a graphene/PMMA film when finished. The film was then transferred to DI water for several times to wash off any residual contamination from the etching process. The rinsed graphene/PMMA film was subsequently scooped up by a holy $\mathrm{Si}_{3} \mathrm{~N}_{4}$ TEM grid (Agar Scientific Y5358), left to dry for $24 \mathrm{~h}$, and then baked at $150^{\circ} \mathrm{C}$ for $15 \mathrm{~min}$ to improve sample adhesion with the grid. Finally, the sample was heated in air at $350^{\circ} \mathrm{C}$ overnight to remove the PMMA scaffold, leaving graphene on the TEM grid.

Transmission Electron Microscopy and Image Processing. Aberration corrected TEM, along with energy dispersive X-ray spectroscopy, was conducted using an FEI Titan 80-300 environmental TEM at $80 \mathrm{kV}$ accelerating voltage. TEM images were processed using Image J. AC-TEM images were adjusted with a bandpass filter (between 100 and 1 pixels) to remove the long range uneven illumination intensity and then smoothed using a Gaussian blur (3-5 pixels). Care was taken to ensure this processing did not influence the interpretation of the original images. The original greyscale TEM images were taken with black atom contrast, then inverted and a false colour (fire) look-up table used to improve the visual contrast. Atomic models based on the TEM images were generated using Accelrys Discovery Studio Visualizer and multislice image simulations of the atomic models were generated using JEMS software with appropriate parameters set to match the conditions of the AC-TEM during imaging. The comprehensive 
experiment and analysis makes it reliable for determination of the structures and time-dependent behaviour of the ordered Si clusters.

\section{Density Functional Theory}

Supporting Information Available: EDX measurements of Si on graphene, measurements of atomic positions and bond lengths.

\section{Acknowledgements}

JHW thanks the Royal Society for support. Part of this work was performed at the Stanford Nano Shared Facilities (SNSF). Part of this work was performed at the Stanford Nano Shared Facilities (SNSF).

\section{Reference}

(1) Bae, S.; Kim, H.; Lee, Y.; Xu, X.; Park, J.-S.; Zheng, Y.; Balakrishnan, J.; Lei, T.; Ri Kim, H.; Song, Y. Il; et al. Roll-to-Roll Production of 30-Inch Graphene Films for Transparent Electrodes. Nat Nano 2010, 5, 574-578.

(2) Blake, P.; Brimicombe, P. D.; Nair, R. R.; Booth, T. J.; Jiang, D.; Schedin, F.; Ponomarenko, L. A.; Morozov, S. V; Gleeson, H. F.; Hill, E. W.; et al. Graphene-Based Liquid Crystal Device. Nano Lett. 2008, 8, 1704-1708.

(3) Zhou, S. Y.; Siegel, D. A.; Fedorov, A. V; Lanzara, A. Metal to Insulator Transition in Epitaxial Graphene Induced by Molecular Doping. Phys. Rev. Lett. 2008, 101, 86402.

(4) Elias, D. C.; Nair, R. R.; Mohiuddin, T. M. G.; Morozov, S. V; Blake, P.; Halsall, M. P.; Ferrari, A. C.; Boukhvalov, D. W.; Katsnelson, M. I.; Geim, A. K.; et al. Control of Graphene's Properties by Reversible Hydrogenation: Evidence for Graphane. Sci. 2009, $323,610-613$.

(5) Wang, Y.; Huang, Y.; Song, Y.; Zhang, X.; Ma, Y.; Liang, J.; Chen, Y. RoomTemperature Ferromagnetism of Graphene. Nano Lett. 2008, 9, 220-224.

(6) Novoselov, K. S.; Fal'ko, V. I.; Colombo, L.; Gellert, P. R.; Schwab, M. G.; Kim, K. A Roadmap for Graphene. Nature 2012, 490, 192-200. 
(7) Arsat, R.; Breedon, M.; Shafiei, M.; Spizziri, P. G.; Gilje, S.; Kaner, R. B.; Kalantarzadeh, K.; Wlodarski, W. Graphene-like Nano-Sheets for Surface Acoustic Wave Gas Sensor Applications. Chem. Phys. Lett. 2009, 467, 344-347.

(8) Warner, J. H.; Rümmeli, M. H.; Bachmatiuk, A.; Wilson, M.; Büchner, B. Examining CoBased Nanocrystals on Graphene Using Low-Voltage Aberration-Corrected Transmission Electron Microscopy. ACS Nano 2010, 4, 470-476.

(9) Warner, J.; Lin, Y.; He, K.; Koshino, M.; Suenaga, K. Stability and Spectroscopy of Single Nitrogen Dopants in Graphene at Elevated Temperatures. ACS Nano 2014, 11806 11815 .

(10) Yang, Z.; Yin, L.; Lee, J.; Ren, W.; Cheng, H.-M.; Ye, H.; Pantelides, S. T.; Pennycook, S. J.; Chisholm, M. F. Direct Observation of Atomic Dynamics and Silicon Doping at a Topological Defect in Graphene. Angew. Chem. Int. Ed. Engl. 2014, 53, 8908-8912.

(11) Sevinçli, H.; Topsakal, M.; Durgun, E.; Ciraci, S. Electronic and Magnetic Properties of \$3d\$ Transition-Metal Atom Adsorbed Graphene and Graphene Nanoribbons. Phys. Rev. B 2008, 77, 195434.

(12) Krasheninnikov, A. V; Lehtinen, P. O.; Foster, A. S.; Pyykkö, P.; Nieminen, R. M. Embedding Transition-Metal Atoms in Graphene: Structure, Bonding, and Magnetism. Phys. Rev. Lett. 2009, 102, 126807.

(13) Robertson, A. W.; Montanari, B.; He, K.; Kim, J.; Allen, C. S.; Wu, Y. A.; Olivier, J.; Neethling, J.; Harrison, N.; Kirkland, A. I.; et al. Dynamics of Single Fe Atoms in Graphene Vacancies. Nano Lett. 2013, 13, 1468-1475.

(14) He, Z.; He, K.; Robertson, A. W.; Kirkland, A. I.; Kim, D.; Ihm, J.; Yoon, E.; Lee, G.-D.; Warner, J. H. Atomic Structure and Dynamics of Metal Dopant Pairs in Graphene. Nano Lett. 2014, 14, 3766-3772.

(15) Zhou, W.; Lee, J.; Nanda, J.; Pantelides, S. T.; Pennycook, S. J.; Idrobo, J.-C. Atomically Localized Plasmon Enhancement in Monolayer Graphene. Nat Nano 2012, 7, 161-165.

(16) Lee, J.; Zhou, W.; Pennycook, S. J.; Idrobo, J.-C.; Pantelides, S. T. Direct Visualization of Reversible Dynamics in a $\mathrm{Si}_{6}$ Cluster Embedded in a Graphene Pore. Nat. Commun. 2013, 4, 1650.

(17) Zhao, J.; Deng, Q.; Bachmatiuk, A.; Sandeep, G.; Popov, A.; Eckert, J.; Rümmeli, M. H. Free-Standing Single-Atom-Thick Iron Membranes Suspended in Graphene Pores. Sci. 2014, 343 , 1228-1232.

(18) Shi, Y.; Kim, K. K.; Reina, A.; Hofmann, M.; Li, L.-J.; Kong, J. Work Function Engineering of Graphene Electrode via Chemical Doping. ACS Nano 2010, 4, 2689-2694. 
(19) Farmer, D. B.; Golizadeh-Mojarad, R.; Perebeinos, V.; Lin, Y.-M.; Tulevski, G. S.; Tsang, J. C.; Avouris, P. Chemical Doping and Electron-Hole Conduction Asymmetry in Graphene Devices. Nano Lett. 2008, 9, 388-392.

(20) Kim, N.; Kim, K. S.; Jung, N.; Brus, L.; Kim, P. Synthesis and Electrical Characterization of Magnetic Bilayer Graphene Intercalate. Nano Lett. 2011, 11, 860-865.

(21) Zhan, D.; Sun, L.; Ni, Z. H.; Liu, L.; Fan, X. F.; Wang, Y.; Yu, T.; Lam, Y. M.; Huang, W.; Shen, Z. X. FeCl3-Based Few-Layer Graphene Intercalation Compounds: Single Linear Dispersion Electronic Band Structure and Strong Charge Transfer Doping. $A d v$. Funct. Mater. 2010, 20, 3504-3509.

(22) Bangert, U.; Pierce, W.; Kepaptsoglou, D. M.; Ramasse, Q.; Zan, R.; Gass, M. H.; Van den Berg, J. A.; Boothroyd, C. B.; Amani, J.; Hofsäss, H. Ion Implantation of GrapheneToward IC Compatible Technologies. Nano Lett. 2013, 13, 4902-4907.

(23) Robertson, A. W.; Allen, C. S.; Wu, Y. A.; He, K.; Olivier, J.; Neethling, J.; Kirkland, A. I.; Warner, J. H. Spatial Control of Defect Creation in Graphene at the Nanoscale. Nat Commun 2012, 3, 1144.

(24) Huang, P. Y.; Ruiz-Vargas, C. S.; van der Zande, A. M.; Whitney, W. S.; Levendorf, M. P.; Kevek, J. W.; Garg, S.; Alden, J. S.; Hustedt, C. J.; Zhu, Y.; et al. Grains and Grain Boundaries in Single-Layer Graphene Atomic Patchwork Quilts. Nature 2011, 469, 389392.

(25) Meyer, J. C.; Kisielowski, C.; Erni, R.; Rossell, M. D.; Crommie, M. F.; Zettl, a. Direct Imaging of Lattice Atoms and Topological Defects in Graphene Membranes. Nano Lett. 2008, 8, 3582-3586.

(26) Robertson, A.; Warner, J. Atomic Resolution Imaging of Graphene by Transmission Electron Microscopy. Nanoscale 2013, 5, 4079-4093.

(27) Wu, Y. A.; Fan, Y.; Speller, S.; Creeth, G. L.; Sadowski, J. T.; He, K.; Robertson, A. W.; Allen, C. S.; Warner, J. H. Large Single Crystals of Graphene on Melted Copper Using Chemical Vapor Deposition. ACS Nano 2012, 6, 5010-5017.

(28) Fan, Y.; He, K.; Tan, H.; Speller, S.; Warner, J. H. Crack-Free Growth and Transfer of Continuous Monolayer Graphene Grown on Melted Copper. Chem. Mater. 2014, 26, 4984-4991.

\section{TOC graphic}

